\title{
Features of calculation of steel structures of bridge cranes at variable loads
}

\author{
A. L. Akhtulov ${ }^{1, *}$, O. M. Kirasirov ${ }^{2}$ and M. O. Kirasirov ${ }^{3}$ \\ ${ }^{1}$ Omsk Tank-automotive Engineering Institute, 644098, 14 military town, 119, Cheremushki vil., \\ Omsk, Russia \\ ${ }^{2}$ Limited liability company «Titaniumavto», 2, 3rd roundabout, Omsk, Russia \\ ${ }^{3}$ Omsk State Agrarian University, 644008, 1, Institutskiy Square, Omsk, Russia
}

\begin{abstract}
The article deals with the calculation of metal bridge cranes operating under the influence of variable loads. Requirements to static and dynamic characteristics of mechanisms of load-lifting cranes are caused by specifics of work, features of a design and operating conditions of the cranes working at variable loadings. Thus, it is proposed to perform calculations of parts of crane mechanisms for endurance, operating under non-stationary variable load, based on the principle of linear summation of damage, allowing the calculation from the point of view of the load equivalent to the entire range of operating loads.
\end{abstract}

\section{Introduction}

In almost all sectors of the economy are widespread metal structures due to their characteristics [1-4]. Sozdanie effective metal designs are based on a comprehensive consideration of the requirements of exploitation reliability and durability, fabrication and installation, which leads to the need of knowledge of work load, the correct choice of structural forms, use of standardized and harmonized solutions and relevant calculations to create designs [5-7].

Currently, most calculations of metal bridge cranes are performed by the method of allowable stresses under certain load combinations [8. 9]. Such as distributed load from self weight of the bridge structure of a crane, point loads from the cargo truck with the load located at mid-spans, concentrated load from a cargo truck with load located at the end of the beam or in dangerous cross-section, i.e. the transition section of the bridge beams from high to low [10].

The sections of the main beams of bridge cranes are mainly used box section in the form of a rectangular parallelepiped, so it is necessary to ensure the allowable values of the moments of resistance of the rectangular section at different ratios of the size of the belts and walls $[11,12]$.

\footnotetext{
* Corresponding author: ahtulov-al1949@yandex.ru
} 


\section{Formulation of the problem}

Metal structures of bridge cranes are characterized by unsteady loads with varying values of stress amplitudes and asymmetry of the operating cycle [10, 13, 14]. To study the actual loading of bridge cranes in typical operating conditions requires continuous recording of their stress state, which is very laborious. Therefore, evaluation of loading elements of metal structures of bridge cranes get statistical processing of the results obtained [15-18] in the research process, modifying the individual components of the total loading of metallic elements: the force of gravity of a lifted load, the swing angle of the load, the magnitude of the departure of the alternating current, weather loads, followed by summation according to the laws of probability theory [19]. This approach to the study of structural elements is incomparably less time-consuming than a comprehensive study of loading in typical operating conditions. However, the determination of the probabilistic characteristics of individual random loads is also relatively time-consuming, and therefore the calculation method for load combinations has been widely used in crane construction [11].

\section{Theory}

When calculating the structural elements of a bridge crane under unsteady loads, it is necessary to know both the law of changes in stress in time and the effect of this law of changes in stress on fatigue resistance. However, for most elements of metal structures of bridge cranes there are no experimental data on the values of fatigue resistance at nonstationary loads acting on the calculated element. Therefore, the fatigue resistance values experimentally determined under the conditions of stationary loads are used in the calculations, taking into account the influence of changes in overload stresses from the nonstationary loading mode on their endurance limits.

Then the summation of fatigue damage from the action of overload stresses at a constant coefficient of asymmetry of the cycle in the case of a step change in the stress amplitudes can be represented by a linear dependence [15]

$$
\sum_{1}^{i_{0}} \frac{n_{i}}{N_{i}}=a,
$$

where $n_{i}$ - the number of cycles of repetition of stresses $\sigma_{i} ; N_{i}$ - fatigue life, i.e. the number of cycles of repetition of stresses required for failure from fatigue under stress $\sigma_{i}$; $\sigma_{t} / i_{0}$ - the number of steps of change in the amplitude of stresses; $a$ - the value characterizing the properties of the metal in connection with the mode of stress change. Load stages with stress amplitude below the endurance limit do not give a share in the sum of damage, because for them the number of cycles $N_{i}$ on the fatigue curve is infinite and the partial damage is zero. It is obvious that with a sufficiently large number of steps to change the amplitude of the stress $i_{0}$ step diagram can be replaced by a continuous distribution with any degree of accuracy. The linearity of the dependence (1) is determined by the fact that the amount of damage increases uniformly, and for each cycle is $1 / N_{i}$.

A lot of works are devoted to the issues of accumulation of fatigue damages under nonstationary load regimes. However, studies were usually carried out on small samples of processed materials used in the manufacture of machine parts. In comparison with them, the 
work of metal structures of cranes at variable voltages has a number of features such as a significant ductility of structural steels, the nature of the impact of loads. In addition, such structures are made of metal with a preserved rolled surface and have stress concentrators in the form of welds. Therefore, the use of the results obtained in the study of typical samples of machine parts to determine the endurance of welded metal structures requires mandatory experimental confirmation.

\section{Results}

In the works $[5,11,20]$ it is proposed to take into account the accumulation of fatigue damage under non-stationary loading regimes with the help of a linear law, taking the value $a=1$, in [1] the work of welded metal structures under non-stationary loading regimes was additionally subjected to a detailed experimental study.

Thus, the fatigue tests carried out under the most unfavorable two-stage loading confirm that in welded metal structures damages accumulate almost linearly and the law of their accumulation can be expressed in the form $-\sum n_{i} / N_{i}=a$. Adopting the sum of relative longevity $a=1$ contributes to the error allowed for engineering calculations, and in most cases gives a margin for fatigue resistance. In addition, it should be noted that the calculation formula for determining the reduced stress value is under the sign of the radical index of the degree of the fatigue curve $m=3 \div 8$. Therefore, even with the highest value obtained experimentally on welded samples $a=1,5$, for example. $\sqrt[6]{1,5} \approx 1,07$, that is, it differs by less than $10 \%$, overestimating the fatigue resistance in the calculation. Then for welded metal structures expression (1) will take the form

$$
\sum_{1}^{i_{0}} \frac{n_{i}}{N^{i}}=1
$$

The effect of stress overload on the fatigue limit is considered for two-stage loading, which corresponds to the division of the load combinations on the first and second design cases [21-23], i.e., given the gravity of the crane components, the gravity load, horizontal forces of inertia of the crane, the angle of deflection of the load from vertical and find out the influence on the endurance limit $\sigma_{r k}$ stress overload, i.e., voltages greater than $\sigma_{r k}$. As applied to the two-stage loading at overload stresses $\sigma_{n}$ and $\sigma_{\kappa}$ by condition (2) the expression is obtained

$$
\frac{n_{n}}{N_{n}}+\frac{n_{k}}{N_{k}}=1
$$

Applying the logarithm operation to expression (3), we obtain

$$
\lg N_{k}-\lg n_{k}=\lg N_{n}-\lg \left(N_{n}-n_{n}\right) .
$$

Then for samples of the metal bridge crane received the influence of variable loads occur handling stress with PP cycles, secondary fatigue curve is constructed in logarithmic coordinates [24], is parallel to the primary fatigue curve obtained at stationary regime of loading (Fig. 1) [10]. 


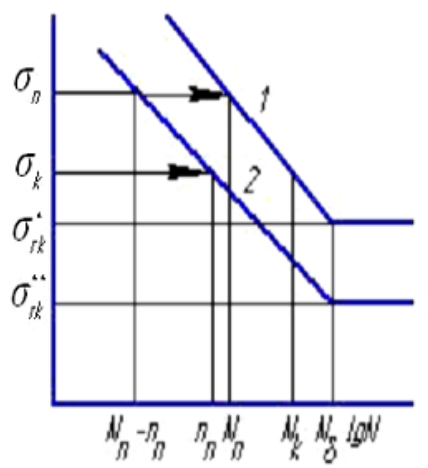

a)

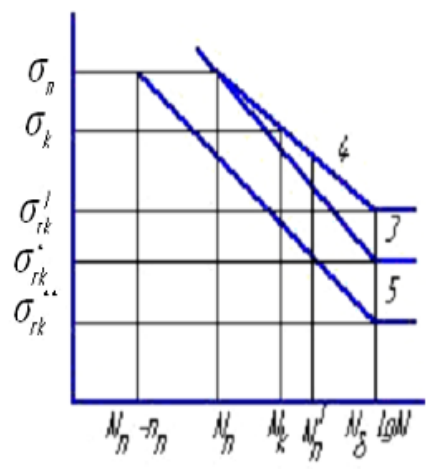

b)

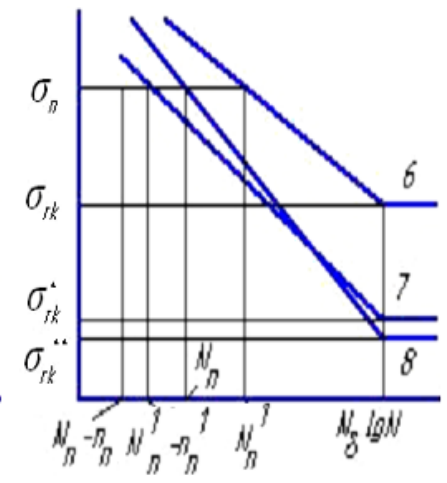

c)

Fig. 1. Graphs of the fatigue dependences: a) with voltages in the place of fatigue crack development $\leq \sigma_{\mathrm{T}}, 1$ - samples in the initial state, 2 - samples after overload and $\sigma_{n}$ in an amount $n_{n}$ cycles'; b) at stresses at the site of fatigue crack development $>\sigma_{\mathrm{T}}, 3$ - samples in the initial state, 4 - samples after static stress overload $a, 5$ - samples after the overload voltage and $\sigma_{n}$ in an amount $\mathrm{n}_{n}$ cycles'; в) at stresses at the site of fatigue crack development $\leq \sigma_{\text {т }}$ at cycle asymmetry coefficient $r-6$ и 7, 8 samples with a coefficient of cycle asymmetry $\mathrm{r}$ after overloading the voltage and $\sigma_{n}$ in an amount $\mathrm{n}_{n}$ cycles at the stress ratio $\mathrm{r}_{1}$

The equations of the primary and secondary fatigue curves can be written as:

$$
\begin{aligned}
& \sigma_{n}^{m} N_{n}=\sigma_{r k}^{m} N_{\sigma}, \\
& \sigma_{n}^{m}\left(N_{n}-n_{n}\right)=\sigma_{r k}^{m} N_{\sigma},
\end{aligned}
$$

then from (5) and (6) we get

$$
\sigma_{r k}^{*}=\sigma_{r k} \sqrt[m]{1-\frac{n_{n}}{N_{n}}}
$$

From the expression of the primary fatigue curve

$$
N_{n}=\frac{\sigma_{r k}^{m} N_{\sigma}}{\sigma_{n}^{m}}
$$

Substituting the value of $N_{n}$ from (8) to (7), we obtain a reduced endurance limit as a result of cyclic stress overload $\sigma_{n}$ in an amount $n_{n}$ cycles equal to

$$
\sigma_{r k}^{*}=\sigma_{r k} \sqrt[m]{1-\left(\frac{\sigma_{n}}{\sigma_{r k}}\right)^{m} \frac{n_{n}}{N_{\sigma}}}=\sigma_{r k} \sqrt[m]{1-\mathrm{A}^{m} \mathrm{~B}}=\varphi_{c} \sigma_{r k},
$$

where $A=\sigma_{n} / \sigma_{r k}$ - the relative magnitude of the overload; $B=n_{n} / N_{\sigma}$ - the relative duration of the overload; $\varphi_{n}$ - coefficient of reduction of endurance limit.

Then, if the metal structures experience overload stresses repeatedly over the entire period of loading cycles, then

$$
\sigma_{r k}^{*}=\sigma_{r k} \sqrt[m]{1-\sum_{1}^{i_{0}}\left(\frac{\sigma_{i}}{\sigma_{r k}}\right)^{m} \frac{n_{i}}{N_{\sigma}}}=\sigma_{r k} \sqrt[m]{1-\sum_{1}^{i_{0}} A_{i}^{m} B_{i}} .
$$




\section{Summary and conclusions}

Thus, in the case where $A^{m} B \geq 1$ or $\sum_{1}^{i_{0}} A_{i}^{m} B_{i} \geq 1$ there will be a destruction of the integrity of metal structures. At the site of the fatigue crack, the amount of residual stresses and stresses from the external load exceeds the yield strength of the metal material and as a result of the action of variable loads, the residual stresses are reduced.

During cyclic overloading of structures stresses $\sigma_{n}$ in an amount $n_{n}$ the secondary fatigue curve (Fig. 1b) will be parallel to the primary curve, but with a changed value of residual stresses.

Then the value of the reduced endurance limit can be determined by the formula:

$$
\sigma_{r k}^{* *}=\sigma_{r k}^{\prime} \sqrt[m]{1-\left(\frac{\sigma_{n}}{\sigma_{r k}^{\prime}}\right)^{\prime} \frac{n_{n}}{N_{\sigma}}},
$$

the law of accumulation of fatigue failure of metal structures of the crane can be expressed by linear dependence

$$
\sum_{1}^{i_{0}} \frac{n_{i}}{N_{i}^{\prime}}=1
$$

where $N_{i}$ - durability of elements of metal structures with the changed value of residual stresses [25].

Thus, the operating loads and stresses arising in the structural elements of the bridge crane, in most cases, are random functions of time, and the characteristics of the fatigue resistance of the structure (service life, endurance limit) are random variables that are characterized by significant dispersion, so the refined methods of calculating fatigue strength are based on probability theory and mathematical statistics.

\section{References}

1. Kudryavtsev I V and Naumenko N E 1976 Fatigue of welded structures (Moscow: Mechanical Engineering) p 270

2. Ryakhin V A and Moshkarev G N 1984 The durability and stability of welded structures for construction and road machines (Moscow: Mechanical Engineering) $\mathrm{p}$ 232

3. Sokolov S A 2005 Metal structures of lifting and transport machines (Moscow: Polytechnic) p 424

4. Sokolov S A 2012 Structural mechanics and metal structures of machines (Moscow: Polytechnic) p 424

5. Serensen S V, Kulaev V P and Nadarevic R M 1975 Bearing capacity and calculations of machine parts for strength (Moscow: Mechanical Engineering) p 488

6. Antulov A L, Ivanova L A, Kirasirov M O and Kirasirov O M 2019 Application of the Substructure Method to Assess the Vibration State of the Bridge Crane Proceedings of 14th International Conference on Electromechanics and Robotics “Zavalishin's Readings”, Smart Innovation, Systems and Technologies 154 Chapter 62 pp 1-11 (DOI:10.1007/978-981-13-9267-2_62) 
7. Antulov A L 2018 The algorithm of numerical calculation of constraints reactions in a dynamic system of transport machine AMSD: IOP Publishing: Journal of Physics: Conference Series 944 C 012002 pp 1-12 (DOI: 10/1088/1742-6596/944/1/012002; EID: 2-s2.0-85042282003)

8. Shishkov N A 1990 The reliability and safety of load-lifting machines (Moscow: Nedra) p 252

9. Badaguas B T 2012 Safe operation of cranes (Moscow: Alpha Press) p 384

10. Gokhberg M M and Pilipchuk S F 1973 Accumulation of fatigue damages in welded metal structures at single-stage single-stage overload Kiev Automatic welding № 4 pp 35-38

11. Dukelsky A I edited by 1971 Handbook on cranes in 2 volumes vol 1 (Moscow Leningrad: Mechanical Engineering) 399 p.

12. Antulov A L, Cuirassiers O M, Cuirassiers M. O. and Chupin P V 2017 Optimization of choice of parameters span beams for formation of a data Bank in automating the design of bridge cranes / // Basic research № pp

13. Antulov A L 2017 Algorithm of numerical calculation of reactions of constraints in a dynamic system of transport machine Dynamics of systems, mechanisms and machines № 4 Tom. 1 pp 3-13

14. Akhtulov A L, Kirasirov M O, Kirasirov O M and Mashonsky V A 2017 The Construction Of The System Of Automation Of Designing Of Load-lifting Cranes Of Bridge Type European Science and Technology Materials of the XVII international research and practice conference (Munich, June 7th - 8th, 2017) Publishing office Vela Verlag Waldkraiburg - Munich - Germany pp 29-35

15. Dukelsky A I edited by 1973 Handbook on cranes in 2 volumes vol 2 (Moscow Leningrad: Mechanical Engineering) p 399

16. Klykov N A 1984 Calculation of fatigue resistance characteristics of welded joints (Moscow: Mechanical Engineering) p 160

17. Lobov N A 2003 Dynamics of cranes on the track (Moscow: Bauman Moscow state technical University) p 232

18. Spitsyn D N 2009 Dynamics of cranes hard suspension cargo (Moscow: Bauman Moscow state technical University) p 160

19. Grigoriev N I 1964 Load cranes (Moscow - Leningrad: Mechanical Engineering) p 168

20. Grigoriev N I, Larionov V P, Novikov G A and Yakovlev P G 1969 Cold resistance of steels under static and cyclic loading (Moscow: Science) p 94

21. Gafarov G G 2015 Inverse problems of dynamics in group variables Monograph (Moscow: FIZMATLIT) p 120

22. Fedotov P I 2015 Structural mechanics and metal structures of machines (Moscow: publishing ASV) p 200

23. Ushakov N S 1988 Overhead electric cranes (Leningrad: Mechanical Engineering) p 352

24. Braude V I et al 1988 Handbook of cranes in 2 volumes Vol 1 Characteristics of materials and loads. Fundamentals of calculation of cranes, their drives and metal structures (Leningrad: Mechanical Engineering) p 536

25. Kornev A D 2013 Construction cranes and lifting devices Guide (Moscow: Phoenix) p 667 